\title{
Layer Formation and Annihilation in an Immiscible Polymer Blend in Electric and Shear Flow Fields
}

\author{
Yang-Ho NA* ${ }^{*}$, Ayaka Yoshino*, Shinsuke TominAGA**, Hiroshi OrIHARA*i, Seiji UJIIE***, and Tomoyuki NAGAYA ${ }^{* * * *}$ \\ *Department of Applied Physics, Graduate School of Engineering, Hokkaido University, Sapporo 060-8628, Japan \\ ${ }^{* *}$ Department of Applied Physics, Graduate School of Engineering, Nagoya University, Nagoya 464-8603, Japan \\ ${ }^{* * *}$ Department of Applied Chemistry, Faculty of Engineering, Oita University, Oita 870-1192, Japan \\ ${ }^{* * * *}$ Department of Electrical and Electronic Engineering, Faculty of Engineering, Oita University, Oita 870-1192, Japan \\ (Received : January 27, 2006)
}

\begin{abstract}
Simultaneous observation of morphological change and measurement of shear stress in an immiscible polymer blend of a liquid crystalline polymer (LCP) and a methyl phenyl silicone oil (MPS) were carried out in electric and shear flow fields by using a system combining a rheometer and a confocal scanning laser microscope (CSLM). Under shear flow and no electric field a thin MPS layer with low viscosity was formed between two parallel plates of the rheometer, which reduced the apparent viscosity. When subjected to an electric field the layer was broken, resulting in the viscosity increase. The relationship between the morphology and the rheology was studied in detail.
\end{abstract}

Key Words: Immiscible polymer blend / Confocal scanning laser microscope / Layer formation and annihilation /

Electrorheological effect / Blend morphology

\section{INTRODUCTION}

Several immiscible polymer blends were found to exhibit a large electrorheological (ER) effect ${ }^{1)}$; when subjected to an electric field the viscosity increases. Such blends are applied to devices for controlling the torque with an electric field. Some studies have fully explained the ER mechanism. ${ }^{2-7)}$ In immiscible polymer blend ER fluids, it is considered that the viscosity change may be brought about by the morphological change. These blends are composed of a liquid crystalline polymer (LCP) and dimethylsiloxane (DMS); where the LCP is greater than the DMS in viscosity, permittivity, and conductivity. ${ }^{3,8)}$ A similar ER effect was also observed in another blend of urethane-modified polypropylene glycol (UPPG) and DMS. ${ }^{5)}$ These blends are classified into two types. ${ }^{4,6}$ In both types there are small droplets dispersed in the matrix; in one type (type I) the droplets consist of the LCP (or UPPG), while in the other (type II) they consist of the DMS.

The mechanism of the ER effect in the type I blend is considered as follows. ${ }^{2-4,7,9)}$ In the absence of an electric field, the apparent viscosity is low because the droplets can flow easily with sliding over each other in the matrix of DMS with low viscosity. When subjected to an electric field, the droplets

$\dagger$ To whom correspondence should be addressed.

E-mail:orihara@eng.hokudai.ac.jp, Tel:+81 11-706-6639, Fax:+81 11-706-6639 of LCP with high viscosity become elongated along the field due to the electrostriction exerted on the interface between the LCP and DMS, and join to form bridges between the electrodes, resulting in an increase of apparent viscosity. As for the type I blend, the morphological change from a dropletdispersed structure to a network structure observed in a polymer blend has been well studied. ${ }^{10,11)}$

The ER mechanism of the type II blend is quite different from that of the type I. ${ }^{4,6,12)}$ It is considered as follows. Under shear flow the droplets of DMS are largely elongated because the DMS is much lower than the LCP in viscosity, and when the shear rate is high enough the stretched droplets coalesce to form a layer of DMS. The DMS layer reduces the apparent viscosity. By application of an electric field, the layer becomes unstable due to the electrostriction exerted on the interfaces between the LCP and DMS and is destroyed; resulting in the ER effect.

In order to fully confirm the mechanisms proposed above, the direct observation of the structure is necessary. Kimura et al. ${ }^{4,5,13)}$ made a specially designed apparatus in which the blend was placed between a ring-shaped outer electrode and a disk-shaped inner electrode. The gap was $0.5 \mathrm{~mm}$ and the thickness of the sample in the vertical direction was about $0.3 \mathrm{~mm}$. With this apparatus they observed several structural changes related to the ER effect under shear flow and electric 
fields. However, the images were not so clear and they needed the help of illustrations sometimes. The blend was confined to a small space so that the rheological measurements could not be made at the same time as the microscopic observations.

To overcome these difficulties, we observed the structural changes with a confocal scanning laser microscope (CSLM) and measured the shear stress with a rheometer at the same time under shear flow and electric fields. ${ }^{9}$ In the type II blend, however, the structural observation was difficult because the thin layer of DMS was formed parallel to the focal plane of the CLSM. In the present study, we improved our system so that we could observe the cross sections perpendicular to the layer by using object lens with a piezo-actuator. In this paper, we show the results of our observations and stress measurements, and discuss the layer formation/annihilation mechanism for the type II blend.

\section{EXPERIMENTAL}

In this study, a liquid crystalline polymer (LCP) and a methyl phenyl silicone oil (MPS), instead of DMS, were used, because the MPS is a better refractive index-matching fluid with the LCP than the DMS. The LCP was supplied by Asahi Chemical Industry (Shizuoka, Japan) and the MPS (KF-96$20 \mathrm{cSt}$ ) was purchased from Shin-Etsu Chemical (Tokyo, Japan). The procedure of preparation has been described in detail. ${ }^{1,2)}$ The viscosities of LCP and MPS are 315 and $0.45 \mathrm{~Pa} \bullet \mathrm{s}$ at $25^{\circ} \mathrm{C}$, respectively.

We made a blend with LCP and MPS in a weight ratio of $2: 1$. This mixture was prepared by dissolving them in tetrahydrofuran followed by evaporation of the solvent in a vacuum. The blend was sandwiched between a bottom glass plate with an ITO-coated electrode and the rotating metal disk of a rheometer (M10 and RS20, Haake) as shown in Fig. 1. The diameter of the top plate and the gap between the two parallel plates were 20 and $0.2 \mathrm{~mm}$, respectively. Since we used a parallel-plate rotational viscometer and the shear rate depends on its position, the shear rate was defined as the one at the periphery of the upper disk. The shear stress at the edge of the top plate was calculated from the torque by assuming that the fluid is Newtonian, though it is a crude approximation in our fluids. The observation was made through the bottom glass plate with a confocal scanning laser microscope (CSLM, Fluoview FV300, Olympus). To observe the structure clearly and to distinguish the LCP and MPS, we doped a small amount of fluorescent dye, IANBD amide (Molecular Probes), to the LCP before mixing the LCP and MPS. It was confirmed that the dye did not dissolve in the MPS. The wavelength of excitation was $488 \mathrm{~nm}$ and a filter of $570 \mathrm{~nm}$ for the detection of light was used. Electric fields were applied to the blend by a synthesizer (model 1940, NF Electric Instruments) and a high voltage amplifier (model 609C-6, Trek). An AC electric field of $10 \mathrm{~Hz}$ was applied to avoid the migration of ionized LCP droplets.

In order to observe the cross sections of the MPS layer, which was formed parallel to the glass plate, we used objective lens with a piezo-actuator moving vertically. Figure 2 depicts the scanning area. The size is $353.6 \mu \mathrm{m}(\mathrm{x}$ or $\mathrm{y}) \times 50 \mu \mathrm{m}(\mathrm{z})$. Let us explain here the scanning method of the $x-z$ plane. We applied a triangular voltage to the piezo-actuator so that the objective lens or the focal plane of the microscope periodically moved up and down, and repeatedly made one-dimensional scan with the CSLM in the $\mathrm{x}$ direction, which was

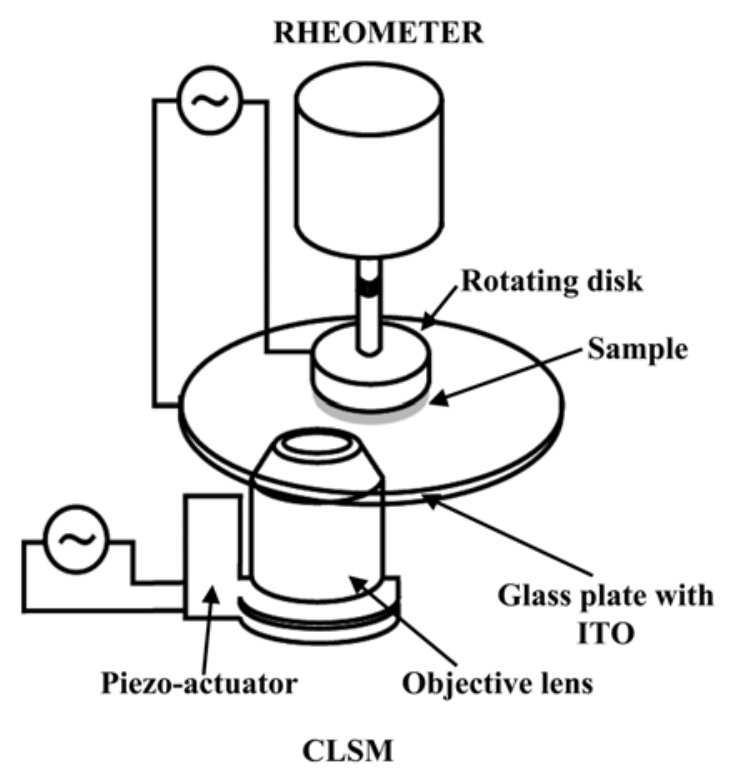

Fig. 1. Schematic illustration of the system combining a CSLM and a rheometer.

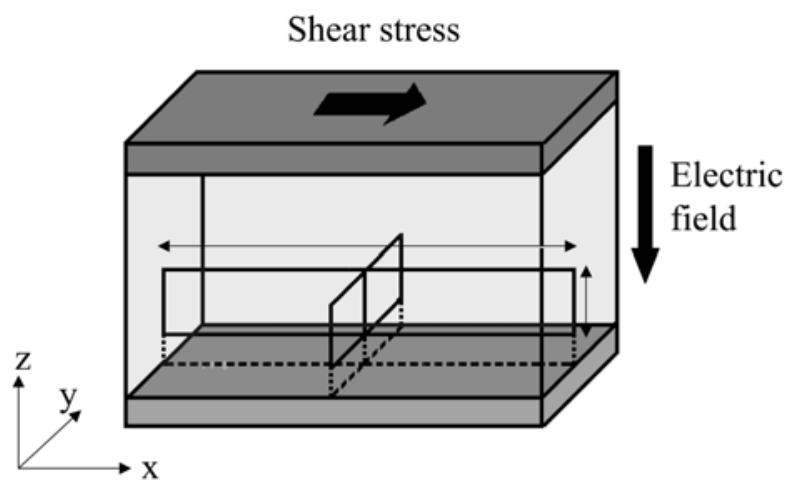

Fig. 2. Scanning area applied in this study. 
synchronized with the oscillation of the objective lens by adjusting the frequency of the triangular voltage. In our experiment, the frequency was set at $4.9505 \mathrm{~Hz}$. Therefore, it took about $0.1 \mathrm{~s}$ to capture one image.

\section{RESULTS AND DISCUSSION}

First, we show the layer formation process after applying a step shear flow of $24 \mathrm{~s}^{-1}$ without electric field in Fig. 3 (a) (x-z plane), (b) (y-z plane) and the corresponding shear stress in Fig. 3 (c). Before applying the step shear flow, we applied a shear of $24 \mathrm{~s}^{-1}$ and an electric field of $4 \mathrm{kV} / \mathrm{mm}$ for $3 \mathrm{~min}$ and waited for $30 \mathrm{~s}$ under no shear flow and no electric field to make a MPS-droplet dispersed structure as shown in Fig. 3(a-1), where the dark region is the MPS. The CSLM images of Fig. 3(a) were observed about $1 \mathrm{~mm}$ far from the edge of the rotating disk and at $\mathrm{z}=0-50 \mu \mathrm{m}$, where the origin of $\mathrm{z}$ was taken at the bottom glass surface, and those of Fig. 3(b) at
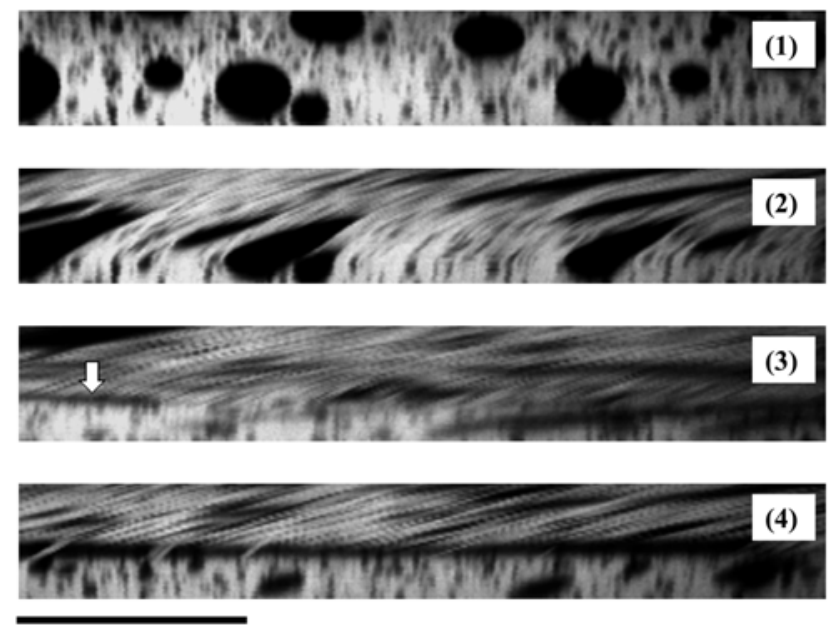

$100 \mu \mathrm{m}$

(a)
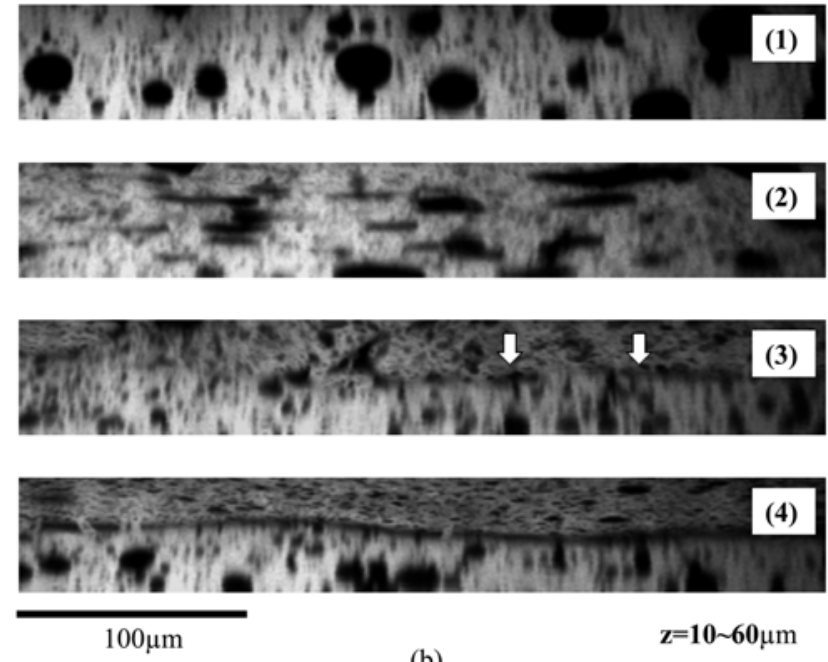

(b) $\mathrm{z}=1-60 \mu \mathrm{m}$, respectively. Note that these observations were made separately and the time dependence of the stress in Fig. 3(c) corresponds to Fig. 3(b). Immediately after the step flow was exerted on the sample, the droplets of MPS became largely elongated because the viscosity of the MPS is much lower than that of the LCP. After a little while the fragments of a dark stripe were formed (an arrow in Fig. 3(a-3)), expanded and coalesced, followed by the formation of a MPS layer (a horizontal thick dark line) in Fig. 3(a-4). The layer works as a slider between the upper and the lower regions. One may notice in Fig. 3(a-4) that the structure above the layer is tilting but not below it. This may be explained as follows. Once a layer was formed, the shear flow was confined to the low viscosity layer, and therefore the LCP below it was fixed to the glass plate, while the upper LCP was moving together with the upper rotating plate. Since the scanning speed was lower than the moving speed of the upper LCP, it apparently looked tilting, though there was almost no shear deformation in it. We could observe similar structural changes in the $y-z$ plane of Fig. 3(b). In this plane it should be noted that the spherical droplet looked oblate when it moved perpendicular to the plane due to the slow scanning speed. It was seen that the formed layer was curved (Fig. 3(b-4)) and furthermore the height of the layer changed irregularly. Corresponding to the structural change the shear stress decreased as shown in Fig. 3(c). However, the stress decrease was almost over before

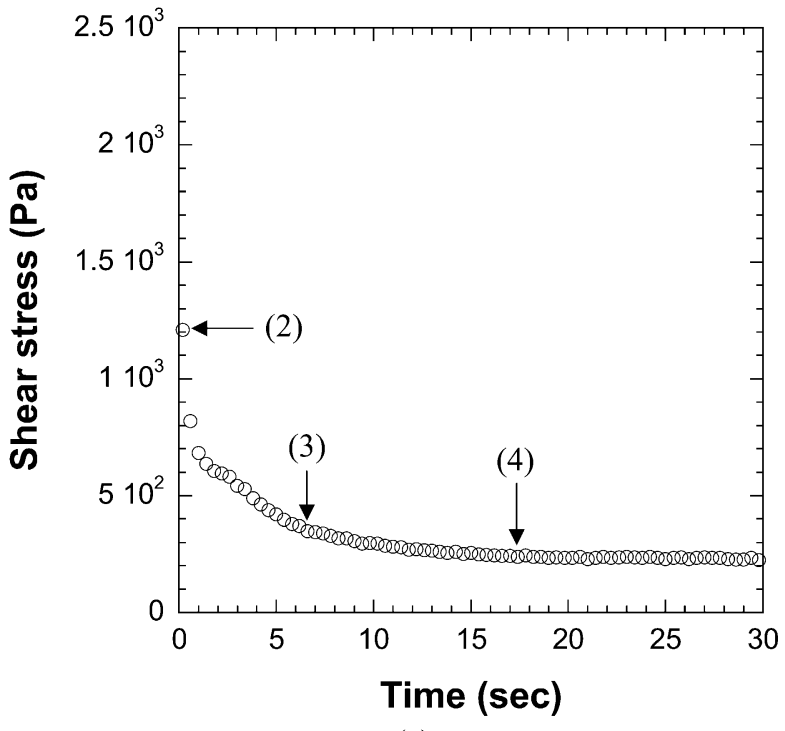

(c)

Fig. 3. Layer formation process of the type II blend in (a) xz plane (1: no shear, $2: 0 \mathrm{~s}, 3: 1.2 \mathrm{~s}, 4: 12.6 \mathrm{~s}$ after the application of shear flow with $24 \mathrm{~s}^{-1}$ ) and (b) yz plane (1: no shear, 2: $0 \mathrm{~s}, 3: 6.6 \mathrm{~s}, 4: 17.4 \mathrm{~s}$ after the application of shear flow with $24 \mathrm{~s}^{-1}$ ). (c) Transient response of shear stress at $24 \mathrm{~s}^{-1}$ corresponding to Fig. 3(b). The bar is $100 \mu \mathrm{m}$ in length. 
the layer was completely formed. This discrepancy may come from the fact that we observed the structure only in a narrow region but measured the stress averaged all over the blend.

Following the above formation process of the layer, the layer annihilation was observed in an electric field. Figures 4(a) and (b) show the structural changes in $x-Z$ and $y-z$ planes, respectively. Both in Figs. 4(a) and (b), the images were observed at $\mathrm{z}=50-100 \mu \mathrm{m}$. The transient response of shear stress corresponding to Fig. 4(b) is shown in Fig. 4(c). In Figs. 4(a) and (b), we applied an AC electric field of $4 \mathrm{kV} / \mathrm{mm}$ for $5.4 \mathrm{~s}$ and $8.2 \mathrm{~s}$, respectively, and then removed it at a constant shear rate of $24 \mathrm{~s}^{-1}$. Before applying the electric field, a dark region corresponding to the MPS layer was seen in Figs. 4(a-1) and (b-1). In Fig. 4(b-1), LCP droplets were observed in the layer. Immediately after applying the field
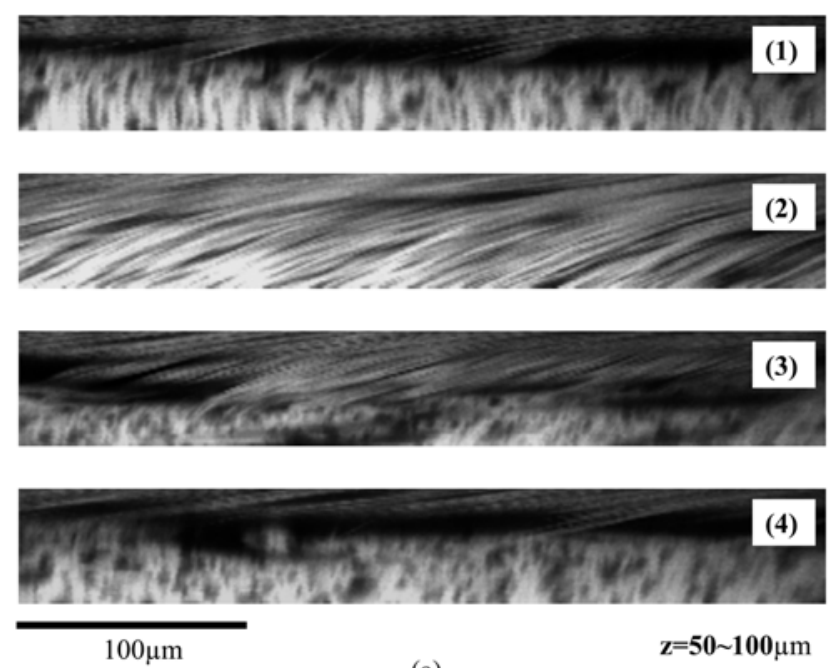

(a)
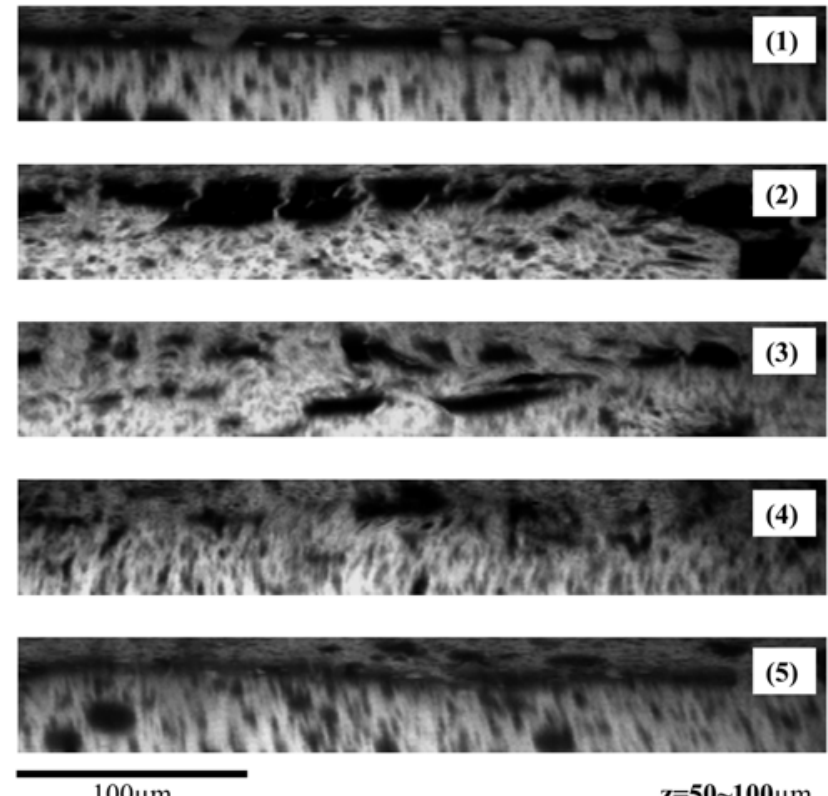

(b)
(Figs. 4(a-2) and (b-2)), the layer began to break. In Fig. 4(b-2), LCP filaments were seen in the layer. In Fig. 4(b-3), the layer almost disappeared. Corresponding to the layer destruction, the shear stress increased as shown in Fig. 4(c). The destruction was thought to be brought about by the Maxwell's electrostriction exerted on the interface between the LCP and MPS. The mechanism may be related to the "Taylor instability". ${ }^{14)}$ It is known that the disintegration of the horizontal interface between a conducting fluid such as water and a non-conducting fluid takes place in a strong vertical electric field. A local vertical displacement of the interface may concentrate the electric lines of force and so increase the vertical force on the interface. If this increase is great enough to counter-balance the pressure drop due to the surface tension, the interface becomes unstable and sometimes jets of the conducting fluid are formed. An analogous phenomenon may occur in our system, because LCP is greater than DMS in permittivity and conductivity. ${ }^{8}$ ) After the removal of the electric field, the layer was gradually formed. Consequently, the shear stress decreased, as previously shown in Fig. 3(c).

We also observed layer annihilation after removing the shear flow without electric field. CSLM images in Fig. 5(a) were observed at $\mathrm{z}=5-55 \mu \mathrm{m}$ and those in Fig. 5(b) at 10-60 $\mu \mathrm{m}$,

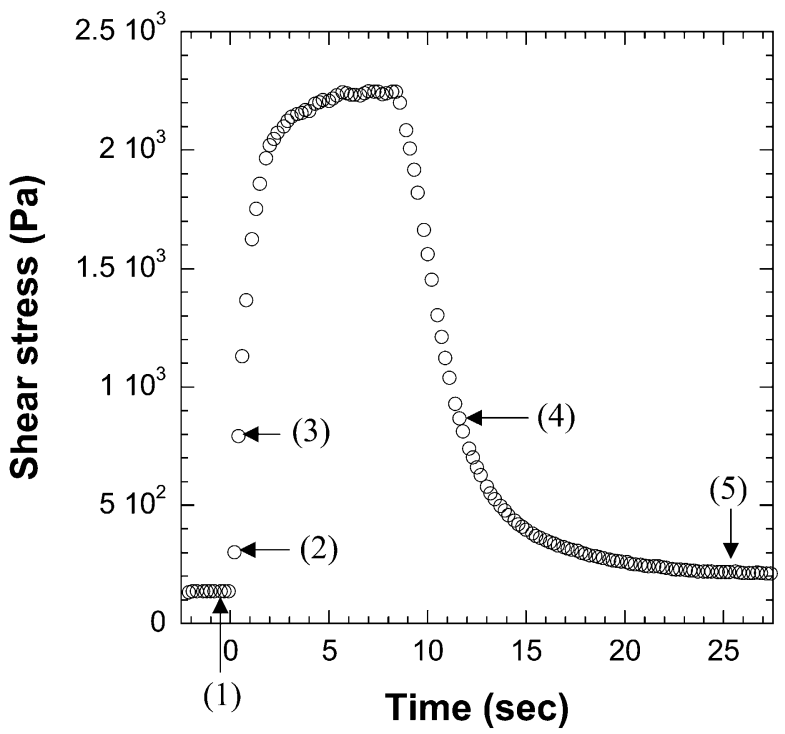

(c)

Fig. 4. Structural changes observed when an electric field of $4 \mathrm{kV} / \mathrm{mm}$ was applied at $t=0 \mathrm{~s}$ and then removed at $5.4 \mathrm{~s}$ and $8.2 \mathrm{~s}$, respectively, for (a) $\mathrm{x}-\mathrm{z}$ plane and (b) $\mathrm{y}-\mathrm{z}$ plane under a constant shear rate of $24 \mathrm{~s}^{-1}$.

(a-1) $t=-1.4 \mathrm{~s}$ before applying the field, (a-2) $t=0 \mathrm{~s}$ immediately after applying the field, (a-3) $t=7.4 \mathrm{~s}$ ( $2 \mathrm{~s}$ later after removing the field), (a-4) $t=8.6 \mathrm{~s}$. (b-1) $t=-0.4 \mathrm{~s}$ before applying the field, (b-2) $t=0.2 \mathrm{~s}$ immediately after applying the field, (b-3) $t=0.4 \mathrm{~s},(\mathrm{~b}-4)$ $t=11.4 \mathrm{~s}(3.2 \mathrm{~s}$ later after removing the field), (b-5) $t=25.4 \mathrm{~s}$. (c) Transient response of shear stress corresponding to Fig. 4(b). 
respectively. After the removal of the shear flow, the upper LCP above the layer made contact with the lower one at several points (Figs. 5(a-2) and 5(b-2)) and the contact regions expanded. It has been pointed out in our previous paper ${ }^{9)}$ that the driving force of the expansion is the interfacial tension between the LCP and MPS, and the annihilation process is similar to the nucleation and growth process of crystals. From this result it is seen that the layer spontaneously annihilates under no shear flow even without electric field.
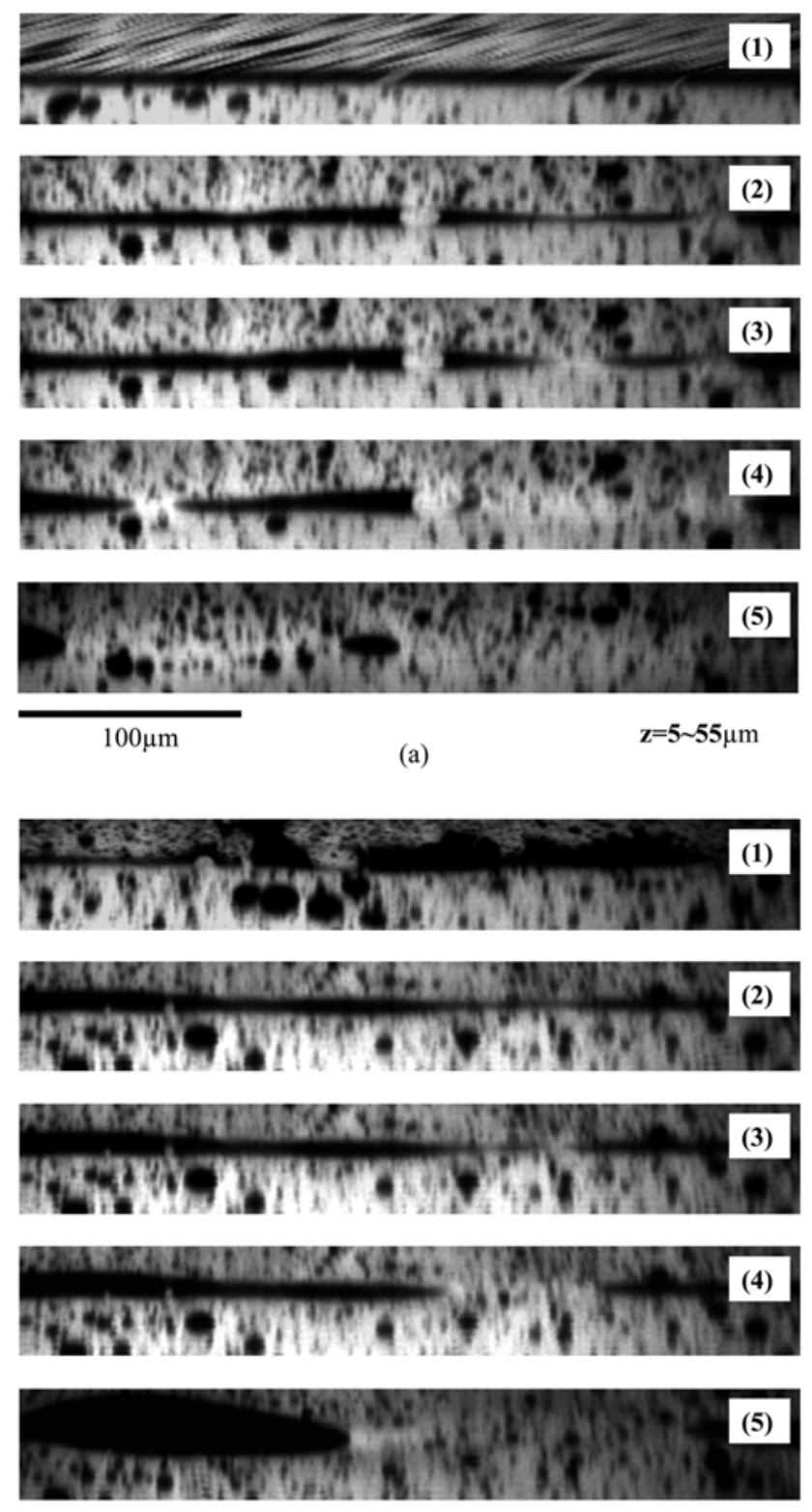

$100 \mu \mathrm{m}$

(b)

$\mathrm{z}=5 \sim 55 \mu \mathrm{m}$

)

Fig. 5. Layer annihilation process after the removal of shear flow in (a) $\mathrm{x}-\mathrm{z}$ plane (1: before removal of shear flow, 2: $4.8 \mathrm{~s}$ later after removing the shear flow, 3: $5.2 \mathrm{~s}, 4: 7.8 \mathrm{~s}, 5: 12.2 \mathrm{~s}$ ) and (b) y-z plane (1: before removal of shear flow, 2: 7.0 s after removing the shear flow, 3: $7.4 \mathrm{~s}, 4: 8.4 \mathrm{~s}, 5: 16.8 \mathrm{~s})$.

\section{CONCLUSIONS}

We investigated structural changes in a type II LCP/MPS immiscible blend with a CLSM modified to observe the cross sections of MPS layer under electric and shear flow fields. Fine structures in the layer such as LCP droplets under no electric field and filaments under an electric field could be observed. The shear stress was measured at the same time and the close relationship between the structure and the viscosity was confirmed in the layer formation and annihilation processes.

\section{Acknowledgments}

The authors would like to thank Dr. A. Inoue of ERtec Co., Ltd. for supplying the LCP. This work has been partially supported by Grant-in-Aid for the 21st Century COE program on "Topological Science and Technology" from the Ministry of Education, Culture, Sport, Science and Technology of Japan.

\section{REFERENCES}

1) Inoue A, Maniwa S, J Appl Polym Sci, 55, 113 (1995).

2) Inoue A, Ide Y, Oda H, J Appl Polym Sci, 64, 1319 (1997).

3) Tajiri $K$, Ohta $K$, Nagaya $T$, Orihara H, Ishibashi $Y$, Doi M, Inoue A, J Rheol, 41, 335 (1997).

4) Kimura H, Aikawa K, Masubuchi Y, Takimoto J, Koyama K, Minagawa K, Rheol Acta, 37, 54 (1998).

5) Kimura H, Aikawa K, Masubuchi Y, Takimoto J, Koyama K, Uemura T, J Non-Newtonian Fluid Mech, 76, 199 (1998).

6) Orihara H, Doi M, Ishibashi Y, Int J Mod Phys B, 13, 1949 (1999).

7) Orihara H, Hosoi Y, Tajiri K, Ishibashi Y, Doi M, Inoue A, $J$ Rheol, 43, 125 (1999).

8) Orihara $\mathrm{H}$, Sugaya $\mathrm{M}$, Tajiri $\mathrm{K}$, Ishibashi $\mathrm{Y}$, Doi M, Inoue $\mathrm{A}, J$ Phys Soc Jpn, 67, 655 (1997).

9) Orihara H, Ikeyama Y, Ujiie S, Inoue A, J Rheol, 47, 1299 (2003).

10) Orihara H, Taki A, Doi M, Inoue A, J Rheol, 45, 1479 (2001).

11) Orihara H, Shibuya T, Nagaya T, Ujiie S, J Phys Soc Jpn, 75, 063802 (2006).

12) Kito $\mathrm{H}$, Tajiri $\mathrm{K}$, Orihara $\mathrm{H}$, Ishibashi $\mathrm{Y}$, Doi $\mathrm{M}$, Inoue $\mathrm{A}$, Int $J$ Mod Phys B, 13, 1983 (1999).

13) Kimura H, Aikawa K, Masubuchi Y, Takimoto J, Koyama K, Uemura T, Int J Mod Phys B, 13, 2001 (1999).

14) Taylor GI, McEwan AD, J Fluid Mech, 22, 1 (1965). 